\title{
Estrategias de mejora según el análisis FODA de un sistema provincial de extensión agrícola
}

\author{
Improvement strategies according to the SWOT analysis of a provincial agricultural extension system \\ Estratégias de melhoria de acordo com análise SWOT de um sistema de extensão agrícola provincial
}

\author{
Rene Antonio Hinojosa Benavides \\ rhinojosa@unah.edu.pe \\ https://orcid.org/0000-0002-0452-3162 \\ Universidad Nacional Autónoma de Huanta, \\ Ayacucho-Perú
}

\author{
Ruggerths Neil de la Cruz Marcos \\ ruggerths.delacruz@unh.edu.pe \\ https://orcid.org/0000-0002-7539-4003 \\ Universidad Nacional de Huancavelica, \\ Huancavelica-Perú
}

\author{
Adelfa Yzarra Aguilar \\ adelfa.yzarra@unh.edu.pe \\ https://orcid.org/0000-0002-6442-6065 \\ Universidad Nacional de Huancavelica, \\ Huancavelica-Perú
}

\author{
Juan Quispe Rodríguez \\ jquispe@unah.edu.pe \\ https://orcid.org/0000-0003-1767-686X \\ Universidad Nacional Autónoma de Huanta, \\ Ayacucho-Perú
}

\section{RESUMEN}

El sistema provincial de extensión agrícola (SIPEA) se caracteriza por elevar la productividad agropecuaria en aras de mejorar la calidad de vida del productor agropecuario, y al no reflejarse esos resultados esperados en las comunidades de la provincia de Acobamba, región Huancavelica, Perú, se abordó la problemática de extensión en el campo agrícola con el objetivo de analizar el SIPEA de dicha provincia, a fin de formular una propuesta que enlace sus componentes en aras de una mejora de la calidad del servicio. Se realizó un análisis situacional de los factores externos e internos, mediante el planeamiento estratégico, así como también se recolectó la información primaria desde agricultores y profesionales, involucrados en los quehaceres agropecuarios; encontrándose como principales fortalezas, debilidades, oportunidades y amenazas, la alta disponibilidad de recursos naturales, el creciente consumo de productos introducidos, el incremento de centros de ventas de productos orgánicos y la importación de productos agrícolas foráneos, respectivamente, concluyendo que, los actores responsables de liderar el SIPEA en Acobamba, realizan actividades de extensión en función a objetivos institucionales, con una débil presencia y pobres resultados, por lo que la magnitud del impacto del SIPEA es insuficiente para la mejora de los niveles productivos de dicha provincia.

Palabras clave: Agropecuaria; Extensión; Planificación estratégica; Análisis FODA
ABSTRACT

RESUMO
The provincial agricultural extension system (PAESY) is characterized by increasing agricultural productivity in order to improve the quality of life of the agricultural producer, and since these expected results are not reflected in the communities of the province of Acobamba, Huancavelica region, Peru, the extension problem in the agricultural field was approached with the objective of analyzing the PAESY of this province, in order to formulate a proposal that links its components in order to improve the quality of the service. A situational analysis of external and internal factors was carried out through strategic planning, as well as the collection of primary information from farmers and professionals involved in agricultural activities; The main strengths, weaknesses, opportunities and threats were found to be the high availability of natural resources, the growing consumption of introduced products, the increase in organic product sales centers and the importation of foreign agricultural products, respectively. The conclusion was that the actors responsible for leading PAESY in Acobamba carry out extension activities based on institutional objectives, with a weak presence and poor results, so the magnitude of PAESY 's impact is insufficient to improve the province's production levels.

Key words: Agriculture; Extension; Strategic planning; SWOT analysis
O sistema de extensão agrícola provincial (SIEAP) caracteriza-se pelo aumento da produtividade agrícola a fim de melhorar a qualidade de vida do produtor agrícola, e como estes resultados esperados não se refletem nas comunidades da província de Acobamba, região de Huancavelica, Peru, o problema da extensão no campo agrícola foi abordado com o objetivo de analisar o SIEAP desta província, a fim de formular uma proposta que ligue seus componentes a fim de melhorar a qualidade do serviço. Uma análise situacional dos fatores externos e internos foi realizada através do planejamento estratégico, assim como informações primárias foram coletadas dos agricultores e profissionais envolvidos em atividades agrícolas; Os principais pontos fortes, fracos, oportunidades e ameaças foram a alta disponibilidade de recursos naturais, o crescente consumo dos produtos introduzidos, o aumento dos centros de venda de produtos orgânicos e a importação de produtos agrícolas estrangeiros, respectivamente, concluindo que os atores responsáveis pela liderança da SIEAP em Acobamba, realizam atividades de extensão de acordo com os objetivos institucionais, com uma presença fraca e maus resultados, de modo que a magnitude do impacto da SIEAP é insuficiente para a melhoria dos níveis produtivos da província.

Palavras-chave: Agropecuária; Extensão; Planejamento estratégico; Análise SWOT 


\section{INTRODUCCIÓN}

El término extensión remonta a la idea de aquello que es complementario, por lo que la extensión agropecuaria conecta lo que aparece como el centro de las pretensiones institucionales y las que se ubican en su entorno, aunque Manchado y Castillo (1) consideran a la extensión como un "territorio abierto, dialogante $y$ en permanente problematización"; en tanto que Tommasino y Cano (2) sintetizaron que la extensión está ligada fuertemente a la difusión cultural, científica y tecnológica; así como también constituye uno de los principales medios para alcanzar el avance de las comunidades rurales hacia situaciones heterogéneas de bienestar (3).

Un programa de extensión agrícola es un instrumento de desarrollo rural dirigido a medianos y pequeños productores con escasos recursos en tierra y capital, con una agricultura orientada a obtener ingresos de la producción agrícola (4). El desarrollo rural implica las acciones conducentes a mejorar la calidad de vida de los habitantes de las zonas rurales (5); así como también el análisis de la fuente de información que acceden los extensionistas es importante para la gestión del conocimiento y la actualización de sus conocimientos técnicos en aras de ofrecer servicios de calidad (6).

En el sector agropecuario las actividades de transferencia de tecnología se conocen como "extensión agrícola", dicho concepto no solo hace referencia al fortalecimiento de variadas tecnologías, sino también a entrenamiento y aprendizaje en general de diversas áreas, por lo que el extensionismo comprende también equipos tecnológicos y conocimiento; y frente a ello debemos recordar que las actividades de extensión agrícola surgieron bajo la idea de mejorar la producción a través de intervenciones de expertos técnicos o investigadores con grupos de productores mediante cursos, talleres y prácticas en campo para la adopción de la tecnología y la puesta en marcha de procesos de innovación, ya que anteriormente la extensión agrícola era un apoyo externo al propio proceso, en la medida en que no involucraba a los productores (7).

La agricultura tiene un gran impacto económico y social, por lo que su dinamismo ha sido propulsado por el importante crecimiento de las exportaciones y el aumento en la producción agropecuaria; sin embargo, se estima que alrededor de tres de cada cuatro agricultores peruanos son pobres, con bajos niveles tecnológicos; además, la propiedad de la tierra se encuentra atomizada, lo que se expresa en el reducido tamaño de la unidad agropecuaria, que está conformada por parcelas pequeñas y dispersas (8); por otro lado, la incidencia del valor del ganado sobre la pobreza es mayor para aquellos que dependen exclusivamente de los ingresos ganaderos y menor para aquellos que tienen tanto las actividades agrícolas y ganaderas (9).

El diagnóstico situacional agropecuario de Acobamba resalta que la mayoría de la población depende de la actividad agrícola y una minoría depende del sector pecuario; los niveles y calidad de la producción agrícola no son óptimos debido a factores meteorológicos 
como temperatura, helada, granizada y sequía); los de manejo que dependen en gran parte de las decisiones que tomen los productores y de los medios que disponen para realizar sus actividades; y los relativos a la variabilidad espacial de los terrenos que tiene un notable efecto para los productores que rentan tierras cultivadas por otros productores o que presentan variabilidad debido a condiciones físicas del suelo y la forma en que se han manejado (10).

Existen instituciones que actúan en el sector agropecuario ya sea por función o por intermedio de proyectos de fortalecimiento de servicios de extensión. La evolución de la producción agropecuaria de Acobamba no es muy alentadora, fundamentalmente por la inadecuada participación de las diferentes instituciones involucradas en este sector, requiriéndose mejorar la calidad de los productos y consolidar las organizaciones de productores para promover alianzas estratégicas entre las instituciones públicas y privadas; concordando con (11) quienes indicaron que la formación de alianzas público-privadas son necesarias para garantizar que la extensión agrícola llegue a todos los agricultores, resaltándose en los nuevos enfoques el planteamiento de una extensión más descentralizada y cercana al terreno. Un servicio de extensión centralizado ha mostrado siempre una dificultad respecto a su pertinencia, debido a su débil capacidad de enfocar la problemática local, sobre todo de los pequeños agricultores (11).
Es importante mencionar que a pesar de que existe el Sistema Provincial de Extensión Agrícola (SIPEA) en la provincia de Acobamba, Huancavelica, se continúe con la baja rentabilidad de sus suelos fértiles, el insuficiente nivel de infraestructura agropecuaria y estancamiento ganadero, por lo que aflora la pregunta de investigación ¿Cuál es la realidad actual del SIPEA en la provincia de Acobamba y que propuestas estratégicas de gestión permiten mejorar este servicio?

Al interior de las comunidades de Acobamba se han constituido los servicios de extensión agropecuaria y organizaciones juveniles que participan constantemente en capacitaciones, a fin de ingresar al mercado con productos agroecológicos competitivos. Estas actividades se realizan conjuntamente con el conocimiento del idioma quechua, propio de Acobamba, como fortaleza de gran utilidad para ganar la confianza de la población rural, a fin de intercambiar información útil, por lo que el propósito del presente estudio fue analizar el SIPEA de dicha provincia, a fin de formular una propuesta que enlace sus componentes en aras de una mejora de la calidad del servicio.

\section{MATERIALES Y MÉTODOS}

Se realizó la planificación estratégica del SIPEA de la provincia de Acobamba, Huancavelica, en la sierra central de Perú, entre los $12^{\circ} 54^{\prime} 17^{\prime \prime}$ de latitud Sur y entre los $69^{\circ} 29^{\prime}$ y $74^{\circ} 31^{\prime} 48^{\prime \prime}$ de longitud Oeste, a una altitud de 3,423 m s.n.m., siendo la agricultura y la ganadería las principales ocupaciones 
de sus habitantes. Los registros narrativos de las actividades del servicio de extensión agrícola se estudiaron mediante la técnica de la observación participante y las entrevistas semiestructuradas a través de un enfoque metodológico cualitativo y participativo, de acuerdo a lo descrito por Sánchez (12), adoptando el siguiente protocolo:

Sondeo estructurado. dirigido a involucrados del sector agricultura (instituciones, profesionales y agricultores), respecto a la percepción de la situación real y sentida del servicio de extensión agrícola (información primaria).

\section{Búsqueda y recopilación de información} publicada. En diagnósticos, censo agropecuario, informes estadísticos, y otros documentos relacionados al servicio de extensión agrícola (información secundaria).

\section{Para realizar el análisis prospectivo de la} realidad agrícola. Se acogió la metodología propuesta por Hernández y Segundo (13) interpretando y analizando la información primaria y secundaria.

\section{Planificación aplicando los resultados} obtenidos en los pasos anteriores. Mediante la metodología del análisis FODA que es una de las principales herramientas estratégicas donde se definen estrategias para el desarrollo de una organización. Esta metodología permitió realizar el análisis a nivel del marco externo e interno del servicio de extensión agrícola.
Definición de la visión, misión y valores del nuevo SIPEA. En talleres de trabajo participativos con los involucrados.

Se definieron objetivos estratégicos. Según los ejes previamente definidos.

\section{Se diseñó la estructura organizativa del sistema} de extensión agrícola. Así como las estrategias y responsabilidades para el monitoreo de su implementación.

Las series de acciones desarrolladas para conseguir el objetivo de la investigación se enmarca en los dos tipos de diseño descrito por Hernández et al.(14) el de Campo, tipo encuesta (para la recolección de datos primarios), donde se realizaron observaciones a través de la aplicación de instrumentos de recolección y análisis de información y el Bibliográfico (para la recolección de datos secundarios), porque se buscó y recopiló información relacionada al tema de trabajo, ambos diseños no experimentales se enmarcan dentro de lo descriptivo transversal, porque se recolectó información en un período determinado.

El marco poblacional estuvo constituido por 293 personas entre jurídicas y naturales, de la que se extrajo una muestra de 197, mediante la siguiente fórmula general de la muestra:

$$
\boldsymbol{n}=\frac{N * Z_{\alpha}^{2} * p * q}{e^{2} *(N-1)+Z_{\alpha}^{2} * p * q}
$$




\section{Donde:}

$\mathrm{N}=293$ (población)

$Z=1,96$ (nivel de confianza de 95\%)

$p=50 \%$ y $q=50 \%$

e $=4 \%$ (error de estimación máximo aceptado)

$\mathrm{n}=$ ? (muestra a determinar)

En la Tabla 1 se muestra la determinación de la muestra igual a 197 y su distribución; dicha asignación se obtuvo mediante el muestreo aleatorio estratificado con afijación proporcional al tamaño del estrato; como ejemplo de dicha afijación muestral se indica que para asignar como muestra a 19 instituciones se dividió la población total de las instituciones, 29 entre la población total de personas jurídicas y naturales, 293 para luego multiplicar dicho resultado por la muestra total definida $=197$, y de igual modo se hizo la afijación proporcional para los 33 profesionales y 145 agricultores.

Tabla 1. Distribución y representatividad de la población y muestra.

\begin{tabular}{lcc}
\hline \multicolumn{1}{c}{ Provincia } & $\mathrm{N}$ & $\mathrm{n}$ \\
\hline Instituciones & 29 & 19 \\
Profesionales & 49 & 33 \\
Agricultores & 2015 & 145 \\
\hline Total & $\mathbf{2 9 3}$ & $\mathbf{1 9 7}$ \\
\hline
\end{tabular}

El tipo de preguntas tuvo su primer paso en la determinación del objetivo de investigación que fue analizar el SIPEA de la provincia de Acobamba en la región Huancavelica, para formular una propuesta que enlace sus componentes en aras de una mejora de la calidad de servicio. Como segundo paso se determinó el método de recolección de datos fue mediante autoadministración. Como tercer paso se determinó el formato de respuesta a las preguntas, que fueron preguntas de escala, a fin de medir la intensidad de las respuestas de los encuestados. Formulando las preguntas de selección para identificar a encuestados calificados, como cuarto paso. Como quinto paso se administró una prueba preliminar a encuestados objetivo del estudio, a fin de identificar malos entendidos o confusión, entre otras reacciones generales de los encuestados, para después preparar el cuestionario e implementar la encuesta.

La información tanto primaria (sondeo) como secundaria (documentos publicados), fue tabulada y procesada mediante la hoja de cálculo Microsoft Excel. La recopilación de las informaciones y de los talleres de trabajo fue sistematizada en el documento final: Planificación Estratégica del SIPEA, en el cual se ordenaron las ideas consensuadas previo análisis y discusión de los actores participantes. 
Realidad actual del SIPEA en la provincia de Acobamba, Huancavelica

En los últimos cuatro lustros el servicio de extensión agraria en Huancavelica y sus provincias fue asumido principalmente por dos sectores: Gubernamental y los Organismos No Gubernamentales (ONGs), ya que hasta antes del año 2000 tradicionalmente la responsabilidad era asumida por el Ministerio de Agricultura a través de sus diferentes órganos desconcentrados, instalados a nivel del departamento de Huancavelica tales como AGRORURAL (antes PRONAMACHS con forestación y conservación de suelos), SENASA (sanidad animal y vegetal), Agencias agrarias provinciales y distritales del MINAG (temas de manejo de cultivos e introducción de nuevas semillas), que formaban parte de las políticas de Estado implementadas a través de proyectos, créditos y fondos rotatorios. Huancavelica no tuvo presencia directa del Instituto Nacional de Investigación Agraria, ni tampoco un organismo generador de nuevas tecnologías acorde a la realidad ambiental de la región Huancavelica.

Solamente después de la recordada "Cabalgata de los Andes" realizada del 02 al 09 de septiembre de 1997 desde Huancavelica hacia Lima, como medida de protesta y exigencia de mayor atención del Estado para Huancavelica, es que recién el gobierno peruano, los gobiernos cooperantes extranjeros, las ONGs, la ONU y Banca Internacional, eligieron a Huancavelica como destino para apoyo prioritario en actividades agropecuarias. La tabla 2 muestra las instituciones que prestan servicio de extensión en Huancavelica, aunándose a otra forma de prestación de servicio de asistencia técnica en el sector agropecuario brindada por profesionales zootecnistas, agrónomos y técnicos agropecuarios de manera independiente, pero los productores tienen que pagar por el servicio que reciben.

Tabla 2. Distribución de instituciones tomadas como muestra.

\begin{tabular}{cll}
\hline $\mathrm{N}^{\circ}$ & \multicolumn{1}{c}{ Provincia/distrito } & \multicolumn{1}{c}{ Instituciones } \\
\hline 1 & Acobamba & Municipalidad provincial \\
2 & & Agencia agraria \\
3 & & AGRORURAL \\
4 & & SENASA \\
5 & & UNH (Escuelas de Agronomía y de Agroindustria) \\
6 & & Cooperativa de ahorro y crédito Huancavelica \\
7 & Andabamba & Municipalidad distrital \\
8 & Anta & Municipalidad distrital \\
9 & & World Vision \\
10 & Caja Espíritu & Municipalidad distrital \\
11 & & Agencia agraria \\
\hline
\end{tabular}




\begin{tabular}{lll}
\hline $\mathrm{N}^{\circ}$ & \multicolumn{1}{c}{ Provincia/distrito } & \multicolumn{1}{c}{ Instituciones } \\
\hline 12 & Marcas & Municipalidad distrital \\
13 & & CARE-PERU \\
14 & Paucará & Municipalidad distrital \\
15 & & Agencia agraria \\
16 & & IESTP “MST” (carrera profesional de Agropecuaria) \\
17 & Pomacocha & Municipalidad distrital \\
18 & & Agencia agraria \\
19 & Rosario & Municipalidad distrital \\
\hline
\end{tabular}

Teniendo en consideración que el funcionamiento del SIPEA es insuficiente, se determina que ello influye en el manejo ineficiente de los recursos disponibles y uso de alternativas agroecológicas, por lo que existe una necesidad de los productores en la adopción de tecnología, innovación y capacitación $(15,16)$. Como señala Landini (17), corresponde repensar las estrategias de acción que involucran el trabajo con grupos de productores asociados y la búsqueda de alternativas para lograr una mayor participación de los beneficiarios en las iniciativas de extensión y desarrollo rural, algo que debe ser contemplado desde el diseño mismo de los proyectos.

$\mathrm{Si}$ bien es cierto que en la actualidad hay varias entidades gubernamentales y no gubernamentales realizando funciones de extensión y asistencia técnica agropecuaria en Huancavelica, también esciertoqueéstassedan de manera no coordinada, cada entidad realiza actividades de acuerdo a sus propios objetivos institucionales, dentro de una duplicidad de actividades y competencia entre ellas, ya que no existe un ente rector y coordinador que defina las zonas de intervención para articularlos, generando desorganización de productores y poco compromiso de trabajo incondicional, como también hay mínima presencia de damas y jóvenes en reuniones de trabajo y la participación de los productores cabezas de familia está condicionada al paternalismo estatal; por tanto, los resultados de impacto y sostenibilidad con adopción de tecnologías no se logran en su totalidad y los niveles de producción agropecuaria del área rural aún siguen bajos.

\section{Oferta del servicio de extensión agraria en Acobamba}

El servicio de extensión agraria en la provincia de Acobamba; Huancavelica es ofertado a través de cuatro sectores principales: Entidades Estatales: lideradas por la Agencia Agraria provincial de la Dirección Regional de Agricultura, en representación del Ministerio de Agricultura y Riego; junto a AGRO RURAL y SENASA. Otras que desarrollan actividades orientadas al sector agropecuario 
son Gerencia Sub Regional, Municipalidades provincial y distritales, la Facultad de Ciencias Agrarias de la Universidad Nacional de Huancavelica con sus escuelas profesionales de agronomía y agroindustria; y el Instituto Superior Tecnológico Público Manuel Scorza Torre, a través de su carrera profesional de producción agropecuaria.

Entidades Privadas: Impulsadas por cooperativas de ahorro y crédito, cajas Municipales y otras empresas privadas dedicadas a la producción y/o venta de materiales y equipos para riego tecnificado, productos agropecuarios, tales como semillas, insecticidas, fertilizantes, pesticidas, entre otros, conjuntamente con la organización de eventos de capacitación sobre temas agropecuarios.

Las Organizaciones no Gubernamentales Las ONGs cumplen actividades basadas en proyectos relacionados a actividades agrícolas, pecuarias y ambientales, sus trabajos están focalizados hacia poblaciones objetivo previamente definidas. A ello se aúnan el Colegio de Ingenieros del Perú, sede Huancavelica, entidades Internacionales como la FAO, Grupo Conjunto Infancia, Nutrición y Seguridad Alimentaria.

Los profesionales y técnicos agropecuarios independientes: quienes por su propia cuenta desarrollan actividades de asistencia técnica hacia los productores, primordialmente en el sector pecuario en temas de sanidad animal, nutrición y reproducción. La particularidad de estos servicios es que son servicios pagados por los usuarios productores.

\section{Análisis FODA}

La Tabla 3 es producto de la sistematización y análisis de los resultados del sondeo ejecutado a las personas naturales y jurídicas, además de la información secundaria. 
Tabla 3. Análisis FODA del sistema provincial de extensión agrícola de Acobamba.

\section{FORTALEZAS (F)}

F1. Disponibilidad de recursos naturales como el suelo, la biodiversidad.

F2. Capacidad instalada para investigación y la generación de tecnologías en Ciencias Agrarias.

F3. Presencia de universidad e instituto tecnológico con profesionales para el sector agropecuario.

F4. Zonas aptas y disponibles para el cultivo de granos, frutas y hortalizas.

F5. Conocimientos y tecnologías andinas conservadas en los productores.

\section{DEBILIDADES (D)}

D1. Creciente consumo de productos de panllevar como arroz, fideos no acordes a la realidad y necesidad.

D2. Escasa difusión de programas con contenido de temas agropecuarios y ambientales

D3. Escasa Información de comportamiento de mercado

D4. Desconocimiento del valor agregado a la poscosecha

D5. Escasa información climática hacia los productores agropecuarios. 
O1. Crecimiento de los mercados de productos orgánicosy de los bioenergéticosy/o anticancerígenos.

O2. Incremento de demanda por servicios de generación, transferencia de tecnología y asistencia técnica por productores agropecuarios.

O3. Existencia de programas estatales de promoción del desarrollo agropecuario.

04. Crecimiento del procesamiento agroindustrial de productos agrícolas.

O5. Libre acceso a sistemas virtuales de información institucional agropecuaria.
F101. Proponer la maximización de los recursos naturales para la diversificación de productos orgánicos .

F2O2. Cubrir la demanda por servicios de transferencia de tecnología proyectando el vínculo de los centros de investigación y sus resultados hacia los productores.

F303. Dinamizar el área agropecuaria informando científicamente, forjando las capacidades de los productores y el vínculo agricultor-investigador.

F404. Definir los volúmenes de producción de productos agrícolas, según la demanda.

F505. Organizar programas de desarrollo agropecuario garantizando el conocimiento integral.
D101. Incrementar las áreas de producción de granos y otros productos nutracéuticos para sustituir importaciones.

D2O2. Diseñar un programa de transferencia tecnológica que corresponda con las necesidades existentes y vincule a los especialistas con los productores en forma integral y decisiva.

D303. Insertarse en un programa que promueva ventajas en el desarrollo agropecuario, comprendiendo mejor el comportamiento de la oferta y la demanda.

D404. Potenciar la utilización de la agroindustria para maximizar la producción y productividad agrícola.

D505. Establecer un programa de información agrícola en tiempo real. 


\begin{tabular}{|c|c|c|}
\hline AMENAZAS (A) & ESTRATEGIAS (FA) & ESTRATEGIAS (DA) \\
\hline
\end{tabular}

A1. La importación de productos agrícolas como producto de competitividad globalizado

A2. Alto precio de insumos agrícolas que incrementan los costos de producción

A3. Efecto negativo de los factores climáticos adversos (sequía, granizada y heladas)

A4. Aumento de la degradación de los suelos

A5. Disminución del tamaño de las unidades agropecuarias, e irregularidades topográficas.
F1A1. Maximizar el uso y conservación de los suelos para obtener mejores productos locales.

F2A2. Promover la investigación y generación de insumos con tecnologías locales.

F3A3. Contactar a los profesionales de la Universidad y/o Instituto tecnológico, para contrarrestar eficaz y eficientemente las adversidades climáticas.

F4A4. Promover el uso racional y rotacional de las diversas zonas aptas para el desarrollo agropecuario.

F5A5. Implementar los conocimientos de los productores sobre tecnologías andinas, por homogeneización del ambiente.
D1A1. Establecer estrategias de fidelización para el retorno de los consumidores locales, y se garantice su lealtad hacia los productos autóctonos.

D2A2. Mejorar y aumentar la difusión de programas que enseñen a disminuir los costos de producción

D3A3. Capacitar e informar a los productores continuamente sobre el comportamiento del mercado influenciado por adversidades climáticas, buscando diferenciación.

D4A4. Construir zanjas para retener el agua y cantidades importantes de suelo, para darles valor agregado.

D5A5. Sensibilizar sobre cambio climático global para afrontar la disminución de unidades de producción agropecuaria. 


\section{RESULTADOS Y DISCUSIÓN}

Resultados del sondeo mediante aseveraciones, realizado a 33 profesionales del agro sobre dificultades del servicio de extensión

\section{La organización insostenible de productores dificulta el servicio de extensión}

En la Figura 1 se observa que el $63,6 \%$ de los 33 profesionales relacionados al sector agrario que fueron encuestados están de acuerdo con esta aseveración y opinan que están de acuerdo en que la organización insostenible de productores dificulta el servicio de extensión y limita la continuidad y sostenibilidad de los trabajos, concordando con GORE-HVCA (18) que señaló que el problema de los agricultores en la provincia de Huancavelica es la baja producción y productividad agropecuaria debido a problemas técnicos, entre los que resalta los insuficientes e inadecuados servicios de información y servicios de sanidad agraria para los productores existiendo una desorganización notable, donde se nota entre ellos un trabajo individualizado sin ningún logro de actividad exitosa a nivel de comunidad.

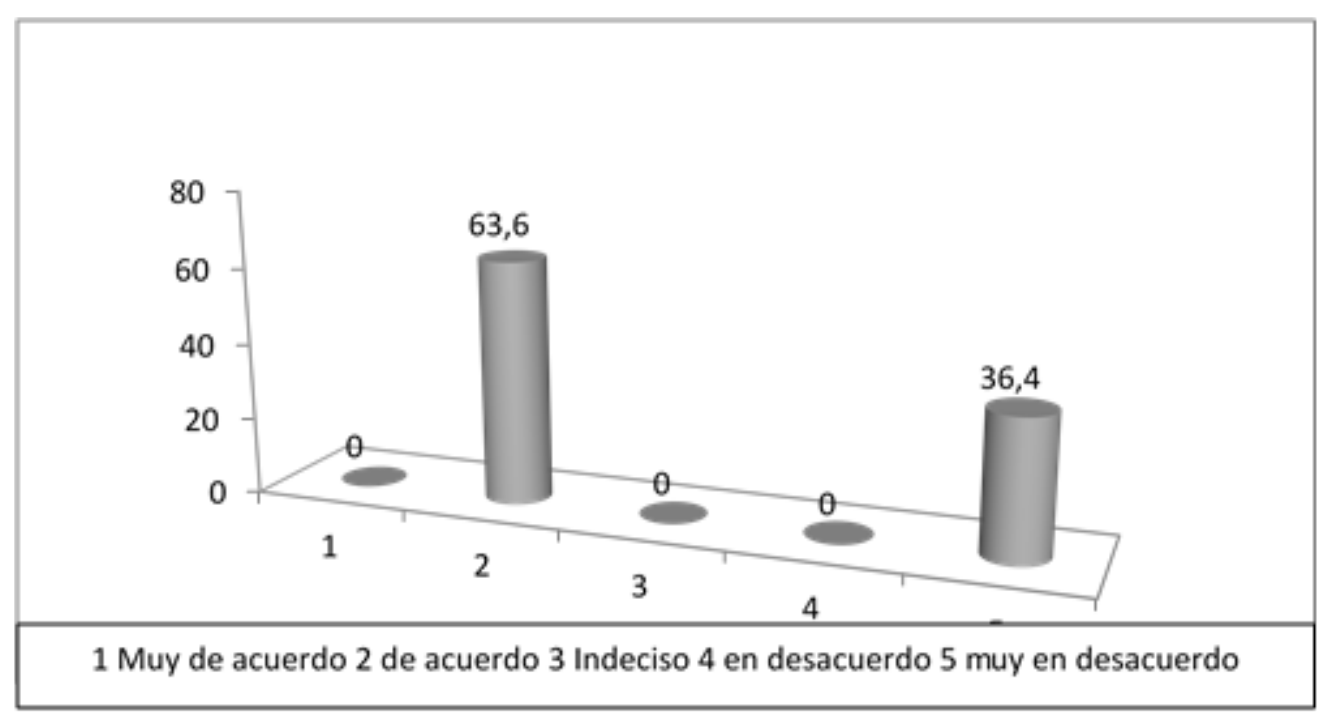

Figura 1. La organización insostenible de productores dificulta el servicio de extensión. Yzarra (19).

La costumbre de beneficiarios sujeto a regalos dificulta el servicio de extensión

En la Figura 2 se muestra que el $69,7 \%$ de los 33 encuestados están de acuerdo con esta aseveración y manifiestan estar muy de acuerdo en que la costumbre de los beneficiarios o productores sujetos a regalos influye negativamente en el servicio de extensión, básicamente para contar con la participación activa de los productores en los procesos de capacitación o charlas, en el cual casi siempre piden alguna recompensa 
material para asistir, con poco interés de participar activamente, tal como lo expresó Barrantes (20) con respecto a las actividades de capacitación y asistencia técnica para la producción de parcelas agroecológicas, ya que este proyecto tuvo un corte asistencialista y en algunas oportunidades fue una dificultad para su aplicación. La costumbre en mención concuerda con una debilidad identificada en el FODA, en el sentido de existir un creciente consumo de productos de panllevar como arroz, fideos no acordes a la realidad $y$ necesidad.

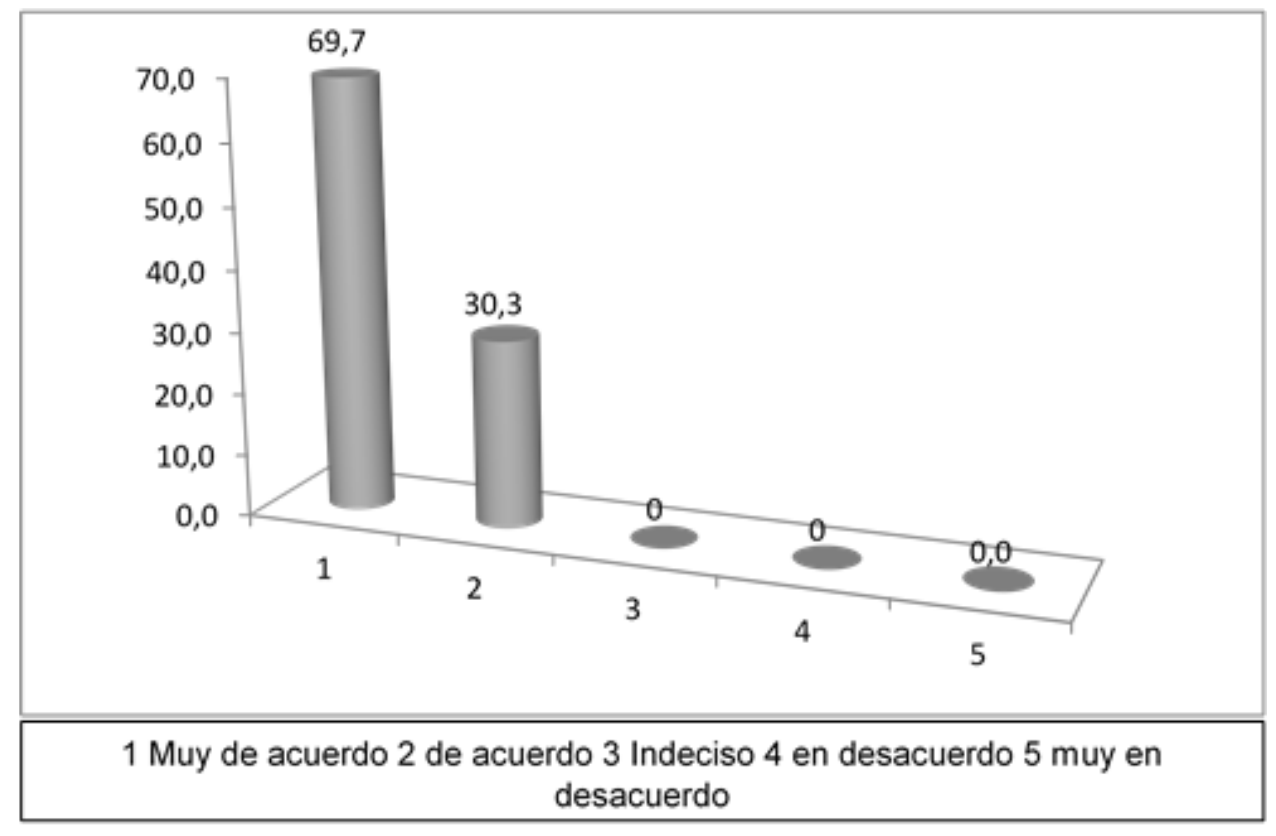

Figura 2. La costumbre de beneficiarios sujeto a regalos dificulta el servicio de extensión. Yzarra (19).

\section{Los programas sociales influyen positivamente en la calidad del servicio de extensión}

La Figura 3 indica que el $69,7 \%$ de los 33 profesionales encuestados no están de acuerdo con esta aseveración y opinan que los programas sociales influyen negativamente en el proceso del servicio de extensión, básicamente porque generan hábitos de dependencia al asistencialismo material, es decir que, para contar con la participación activa de los productores en los procesos de capacitación, es común el tener que dar el inseparable refrigerio, coincidiendo con Barrantes et al. (11) quienes señalaron que un servicio de extensión centralizado no es pertinente debido a su débil capacidad de enfocar la problemática local, sobre todo de los pequeños productores. 


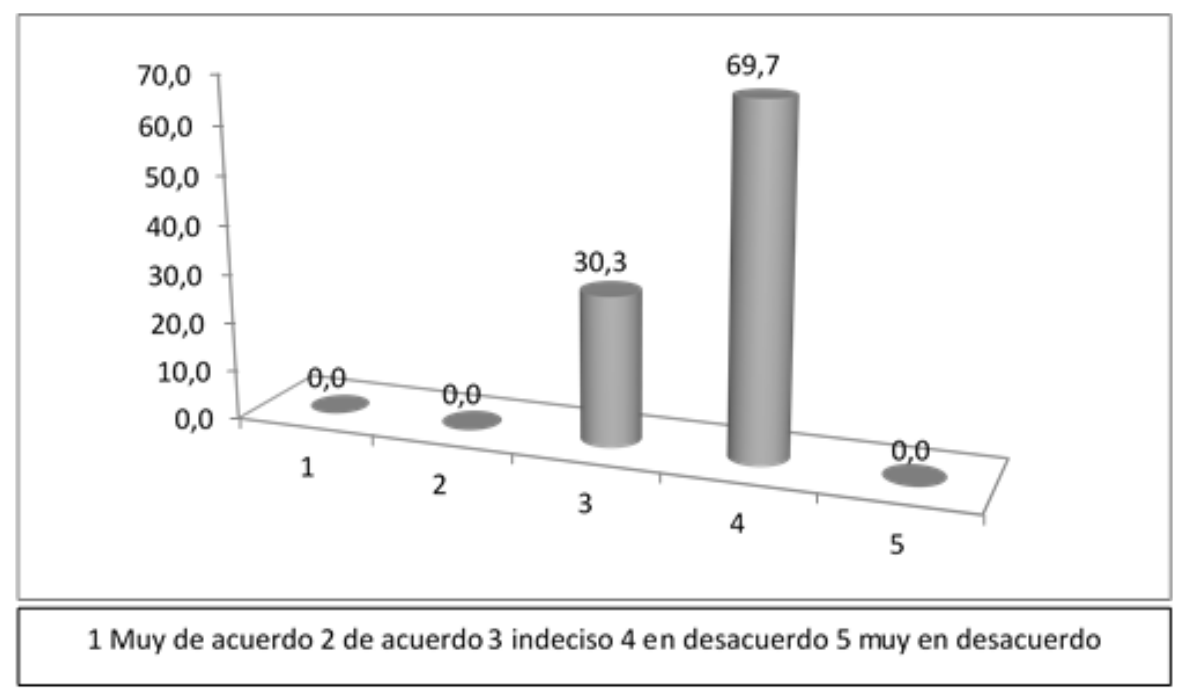

Figura 3. ¿Los programas sociales influyen positivamente en la calidad del servicio de extensión? Yzarra (19).

\section{Resultados del sondeo mediante aseveraciones realizado a 19 instituciones del agro sobre dificultades del servicio de extensión}

\section{La competencia entre instituciones públicas y ONGs dificulta el servicio de extensión}

La Figura 4 indica que el 52,6\% de las 19 instituciones encuestadas está de acuerdo con esta aseveración y afirman que la competencia entre instituciones públicas y ONGs dificulta el servicio de extensión. Todas las instituciones encuestadas concuerdan en pensar que son pocos los productores que muestran interés propio de trabajo por su propio bienestar, tal como lo señala Barrantes (20) en el sentido que se establecen enfrentamientos entre las instancias del Estado y las ONGs, sobre temas y formas de intervención.

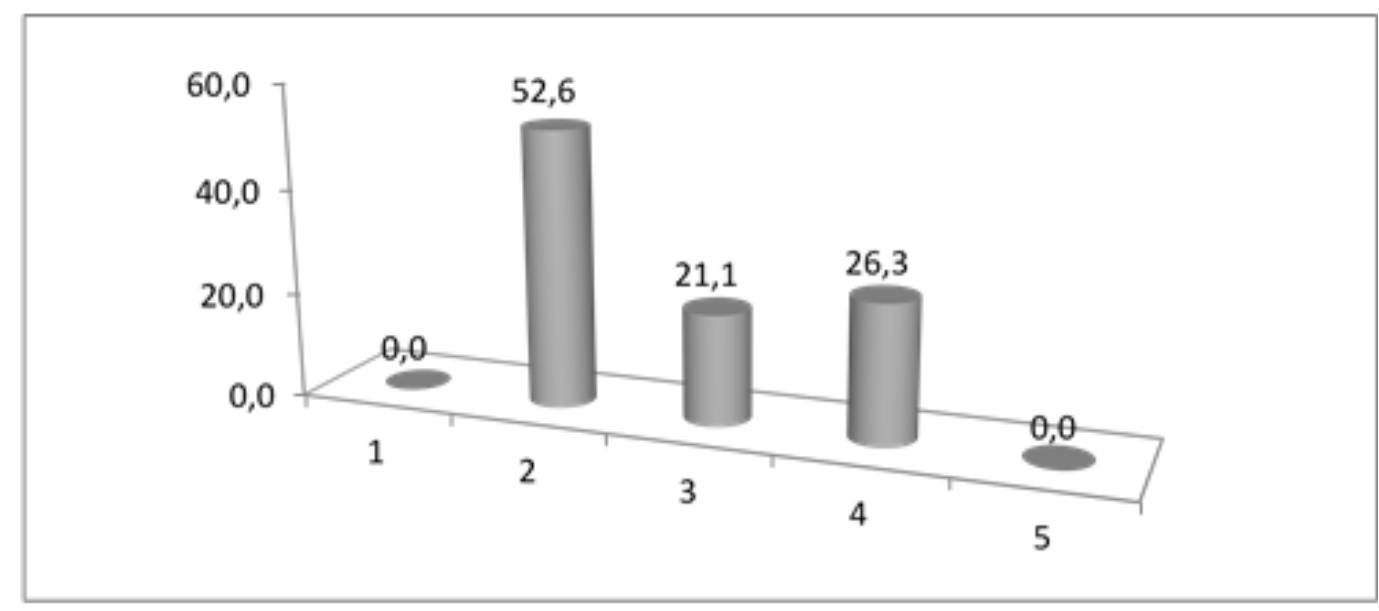

Figura 4. La competencia entre instituciones públicas y ONGs dificulta el servicio de extensión. Yzarra (19). 
Poco compromiso de trabajo incondicional dificulta el servicio de extensión

La Figura 5 muestra que todas las instituciones encuestadas están de acuerdo con esta aseveración y mencionan que son pocos los productores que muestran interés propio de trabajo por su propio bienestar, situación propicia para tener en cuenta a
Landini (17) quien recomienda trabajar con grupos de productores asociados para lograr una mayor participación de los beneficiarios. Este escaso compromiso concuerda con una debilidad identificada en el FODA, en el sentido de existir una escasa información de comportamiento de mercado.

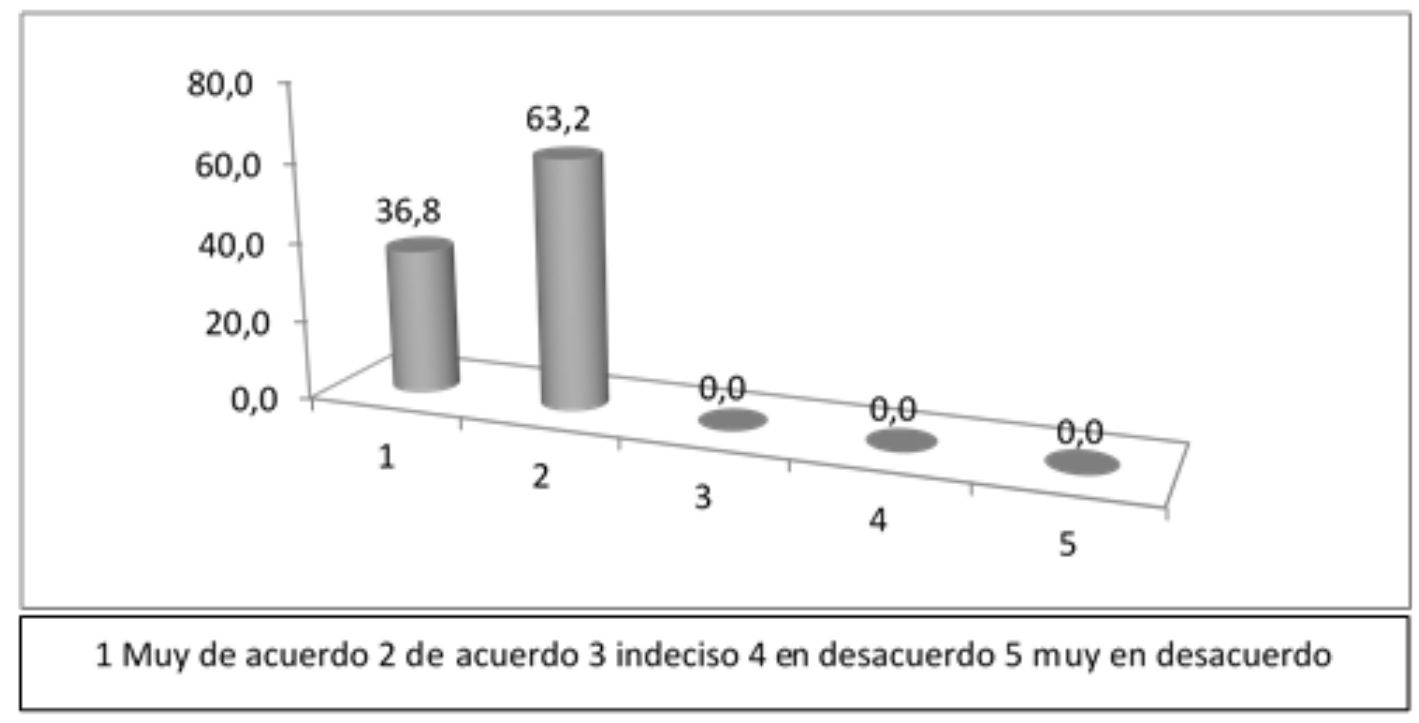

Figura 5. Poco compromiso de trabajo incondicional dificulta el servicio de extensión. Yzarra (19).

Resultados del sondeo mediante aseveraciones realizado a 145 agricultores sobre dificultades del servicio de extensión

\section{El personal de la Agencia Agraria absuelve sus inquietudes referidas a sus problemas agropecuarios}

La Figura 6 indica que el $69 \%$ de los 145 agricultores encuestados no está de acuerdo con esta aseveración y está descontento con el servicio que presta el personal de la agencia agraria de Acobamba, como bien lo señalan Saltos y Lara (21), los agricultores dedicados a la producción de tubérculossemillas deben tener los conocimientos y la experiencia necesaria para proteger sus cultivos; ya que diversas situaciones pueden conllevar a su insatisfacción e inconformidad. Este descontento concuerda con una debilidad identificada en el FODA, en el sentido de un desconocimiento de valor agregado a la poscosecha. 


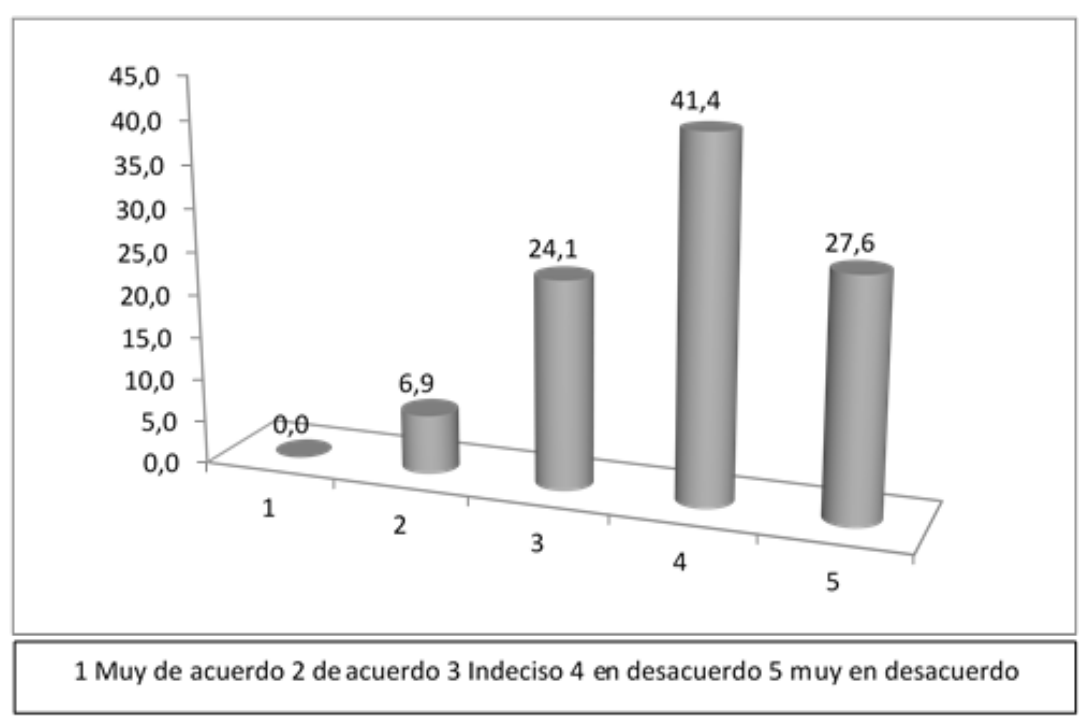

Figura 6. El personal de la agencia agraria absuelve sus inquietudes referidas a sus problemas. Yzarra (19).

Los servicios que presta la agencia agraria Acobamba son en general muy buenos

La Figura 7 muestra que el $80 \%$ de los 145 agricultores encuestados no está de acuerdo con esta aseveración y opinan estar disconformes con los servicios que presta la Agencia Agraria de Acobamba, calificándolos de insuficientes, en concordancia con Espinosa et al. (10) quienes manifestaron que los niveles y calidad de la producción agrícola no son óptimos debido a factores meteorológicos, los de manejo y los relativos a la variabilidad espacial de los terrenos; en concordancia también con Barrantes (20) quien aseveró que las actividades de extensión agrícola en el Perú se manejan a través de entidades gubernamentales y no gubernamentales, pero ninguno de ellas llega óptimamente al pequeño productor, quien constituye la población más importante en países del tercer mundo.

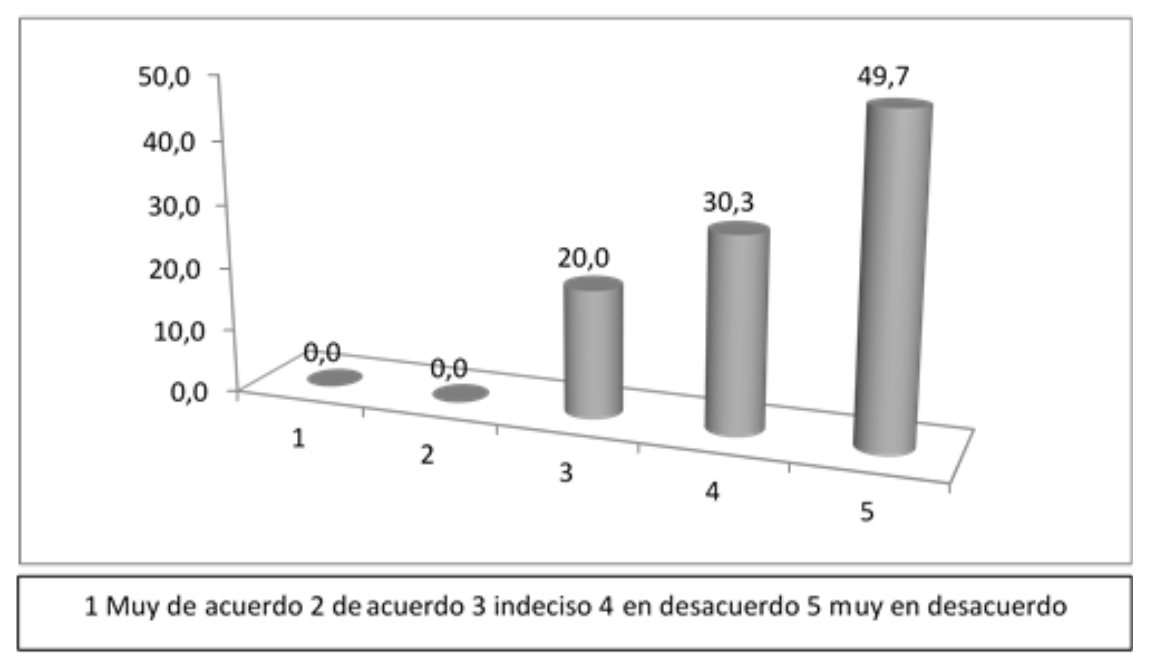

Figura 7. Los servicios que presta la agencia agraria Acobamba son en general muy buenos. Yzarra (19). 


\section{Los servicios de extensión de la Universidad son en general muy buenos}

La Figura 8 indica que el $68,3 \%$ de los 145 agricultores encuestados no está de acuerdo con esta aseveración, y adicionalmente opinan que los servicios de extensión que brinda la Universidad Nacional de Huancavelica a través de sus escuelas profesionales en Acobamba, no son muy significativos, es decir, que la presencia de la universidad en el campo agrario es muy débil. Esta ausencia de la universidad concuerda con una debilidad identificada en el FODA, en el sentido de adolecer de una escasa información climática hacia los productores agropecuarios; ya Licona y Vélez (22) habían manifestado que las funciones sustantivas de la universidad son: investigación, docencia y extensión, cuyo contenido se plantea problemáticas contextuales y valorativas, dinámicamente con relación a la realidad, articulando pilotos con criterios y contrastes del cambio.

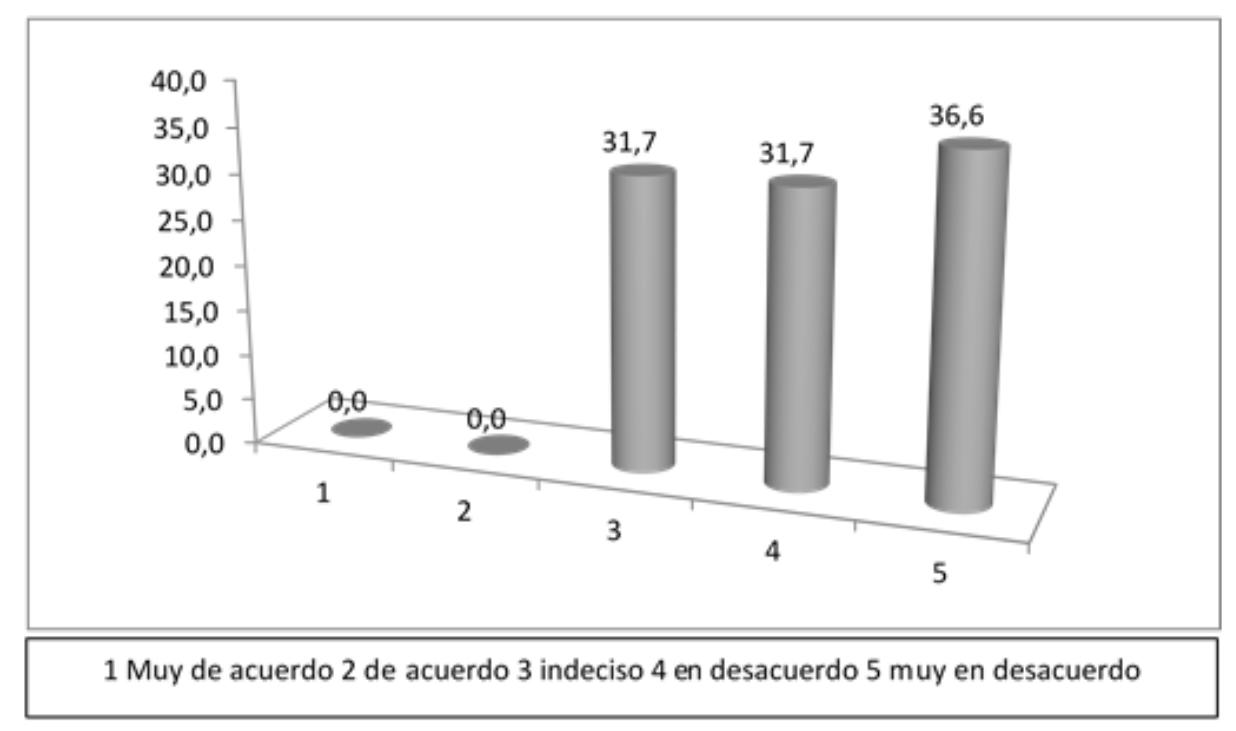

Figura 8. Los servicios de extensión de la Universidad son en general muy buenos. Yzarra (19).

\section{Propuesta agroecológica para una mejor calidad del SIPEA}

A la luz del análisis del SIPEA de Acobamba, Huancavelica, en el marco de las estrategias definidas en su FODA, se formula la siguiente propuesta:

Dinamizar el área agropecuaria con información científica que fortalezca las capacidades de los productores y el vínculo agricultor-investigador, teniendo en cuenta la definición de los volúmenes de producción de productos agrícolas según la demanda, a través de una maximización de los recursos naturales para la diversificación de productos orgánicos, así como también la cobertura de la demanda por servicios de transferencia de tecnología mediante la proyección del vínculo de los centros de investigación y sus resultados hacia los productores, organizando programas de desarrollo agropecuario para garantizar el 
conocimiento integral, sobre todo en cuanto a la asociación de cultivos, ya que se consigue producciones mayores por unidad de superficie cultivada, se aprovecha mejor el agua, hay más sombra, menos evaporación directa y más transpiración a través de las plantas, teniendo muy en cuenta el fortalecimiento organizacional.

Promover la investigación y generación de insumos con tecnologías locales, contactando a los profesionales de la Universidad y/o Instituto tecnológico, para contrarrestar eficaz y eficientemente las adversidades climáticas; considerando que un cambio tecnológico para la repotenciación de cultivos y crianzas constituye el camino más idóneo para una mejor productividad; de igual modo incentivar el uso y conservación de los suelos agrícolas aplicando el manejo integrado de plagas y malezas, conpesticidasnotóxicos, detalmanera que se pueda obtener mejores productos locales, implementando y respetando los conocimientos de los productores sobre tecnologías andinas, promoviendo también el uso racional y rotacional de las diversas zonas aptas para el desarrollo agropecuario, evitando la percolación de nutrientes del suelo que se pueden perder hacia aguas superficiales y subterráneas, y preservando la materia orgánica en el suelo, evitando la erosión.

Incrementar las áreas de producción de granos y otros productos nutracéuticos para sustituir importaciones, insertándose en un programa que promueva ventajas en el desarrollo agropecuario y una mejor comprensión del comportamiento de la oferta y la demanda que contemple un programa de información agrícola en tiempo real provisto de un programa de transferencia tecnológica que corresponda con las necesidades existentes y vincule a los especialistas con los productores en forma integral y decisiva, potenciando de esa manera la agroindustria a fin de maximizar la producción y productividad agrícola. La forma profesional y empresarial de hacer agricultura necesita que los agricultores posean conocimientos innovadores, habilidades, aptitudes y destrezas, porque estas les proporcionan confianza y protagonismo en la solución de sus propios problemas.

Establecer estrategias de fidelización para el retorno de los consumidores locales, y se multipliquen garantizando su lealtad hacia los productos autóctonos, propiciando la mejora y aumento de la difusión de programas que enseñen a disminuir los costos de producción, así como también mediante la capacitación e información continua a los productores sobre el comportamiento del mercado influenciado por adversidades climáticas, buscando diferenciación y también la sensibilización sobre cambio climático global para afrontar la disminución de unidades de producción agropecuaria, construyendo zanjas para retener el agua y cantidades importantes de suelo, para darles valor agregado, ya que en el nuevo sistema de transferencia los agricultores son los principales actores que, adoptan la tecnología recomendada y al mismo tiempo son los comunicadores de los resultados obtenidos a los demás productores.

CONCLUSIONES 
En el sector agrícola y por ende en el servicio de extensión se tienen involucradas a instituciones públicas, privadas y organizaciones de la sociedad civil, caracterizados por realizar trabajos no articulados, en el que los actores responsables de liderar el servicio de extensión agrícola realizan actividades de extensión en función a objetivos institucionales, con una débil presencia y pobres resultados de éxito, por lo que la magnitud del impacto del servicio de extensión agrícola es débil para la mejora de los niveles productivos y para la economía de la población de Acobamba; los cuales pueden subsanarse de forma exitosa si la organización implementa estrategias competitivas que permitan el logro de los objetivos establecidos.

El análisis de la situación actual del servicio de extensión agrícola tanto a nivel regional como provincial, confirma la necesidad de implementar un servicio de extensión con nuevo paradigma, basado en claves como la excelencia en el servicio, anticipación a los futuros sucesos e innovación permanente para llegar a satisfacer las necesidades sentidas de los productores agrícolas, teniendo en cuenta que, la estructura organizativa del SIPEA está constituida por tres subsistemas: el de generación de tecnologías, de información y comunicación agrícola, y el de transferencia de tecnologías; siendo una fuente de autonomía y satisfacción laboral para quienes emprenden un negocio.
1. Manchado $M$, y Castillo RA. extensão será política ou não será. Algumas reflexões sobre as potencialidades e dificuldades de práticas militantes e extensionistas nas prisões santafesinas. Revista Eletrônica da Faculdade de Direito da Universidade Federal de Pelotas 2018;4(1), 245-268

2. Tommasino $\mathrm{H}$, y Cano A. Modelos de extensión universitaria en las universidades latinoamericanas en el siglo XXI: tendencias y controversias. Revista Universidades 2016; 67:7-24

3. Henao A, y Tobasura I. Enfoques de extensión rural para el desarrollo de productores de mora en el departamento de Caldas. Revista Ciencia y Agricultura 2018;15 (2), 25-38

4. Aguilera $R$, y Sacoto V. Propuesta de modernización de los servicios de extensión agropecuaria nuevo paradigma para el desarrollo empresarial sostenible de las comunidades agrícolas en el Ecuador. Revista DELOS 2015;8(23),1-11

5. Cárdenas J, y Vallejo, L. Agricultura y desarrollo rural en Colombia 2011-2013: una aproximación. Apuntes Cenes 2016;35(62):87123

6. Saavedra D, y Briones M. Metodología para analizar los sistemas de extensión agrícola. Fundación para el Desarrollo Tecnológico Agropecuario y Forestal de Nicaragua (FUNICA). Managua, Nicaragua. 2020

7. Amaro M, De Gortari R. Políticas de

\section{REFERENCIAS BIBLIOGRÁFICAS}


transferencia tecnológica e innovación en el sector agrícola mexicano. Rev. Agricultura, Sociedad y Desarrollo 2016;13, 449-471

8. Libelula F. Diagnóstico de la Agricultura en el Perú. Informe final. Peru Opportunity Fund. Lima, Perú., 2011:12,71

9. Paredes R, y Escobar F. El rol de la ganadería y la pobreza en el área rural de Puno. Revista de Investigaciones Altoandinas 2018;20 (1):3960

10. Espinosa J, Palacios E, Tijerina L, Ortiz C, Exebio A, Landeros C. Factores que afectan la producción agrícola bajo riego: cómo medirlos y estudiar su efecto. Revista Tecnología y Ciencias del Agua 2018;9 (2), 175-191

11. Barrantes Ch, Salinas J. y Yagüe L. Factors that influence access to agricultural ad livestock extension in Peru: in search for more inclusive models. Revista Agricultura, sociedad y desarrollo 2017;14 (2):205-217

12. Sánchez A. Constitución Política, acuerdo nacional y planeamiento estratégico en el Perú. Lima, Perú: CEPAL. 2003

13. Hernández $H$, y Segundo, M. Análisis prospectivo de las cooperativas de trabajo asociado. Universidad Externado de Colombia, Bogotá, Colombia. 2004;117

14. Hernández S, Fernández C, y Baptista L. Metodología de la Investigación. 5ta. Edc. México D.F., México: McGraw-Hill. 2010;656

15. Pavón M. Percepción de los agricultores de las formas de extensión en el sector cooperativo y campesino en el municipio San José de las Lajas. In Congreso Científico del INCA, XVII, San José de las Lajas, 2010:22-26. Instituto Nacional de Ciencias Agrícolas. 16. Pérez T.
Propuesta metodológica para el análisis de la seguridad alimentaria a nivel local en Cuba. 2010. [Tesis Doctoral. Universidad Agraria de la Habana, Cuba]. https://n9.cl/329m8

17. Landini F. Problemas Enfrentados por los Extensionistas Rurales Argentinos en el Ejercicio de su Labor desde su Propia Perspectiva. Revista de Economía y Sociología Rural 2013;51(Supl1): 79-100

18. GORE-HVCA. Gobierno Regional de Huancavelica Proyecto: "Mejoramiento del Servicio de Extensión Agraria a los Productores de Frutales en 09 Distritos, 04 Provincias del Departamento de Huancavelica. Perú. 2014.

19. Yzarra A. Planificación estratégica de un sistema provincial de extensión agrícola (SIPEA) para la provincia de Acobamba 2017. [Tesis de Maestría, Universidad Nacional de Huancavelica, Perú]

20. Barrantes $C$. El reto de la extensión agraria en el Perú: de la transferencia de tecnologías a un trabajo integrado sobre el territorio, provincia de Aymaraes. [Tesis doctoral, Escuela Técnica Superior de Ingenieros Agrónomos, Madrid, España]. 2015. https://n9.cl/2n1cp

21. Saltos R, y Lara E. La producción de semillas en la provincia de Bolívar y la importancia del suelo. Alfa, Revista de Investigación en Ciencias Agronómicas y Veterinarias, 2019; 3 (7):15-23

22. LiconaW, $\mathrm{y}$ VélezR. Currículocomprehensivo y extensión universitaria. Experiencia de municipio de San Bernardo, Cundinamarca. Univ. Empresa, 2007;6 (13):59-74 